\title{
Integrating extended focused assessment with sonography for trauma (eFAST) in the initial assessment of severe trauma: Impact on the management of 756 patients
}

\author{
Laurent Zieleskiewicz ${ }^{a, b}, *$, Raphaelle Fresco a, Gary Duclosa, François Antoninia, \\ Calypso Mathieua,c, Sophie Medama , Coralie Vignea, Marion Poiriera, \\ Pierre-Hugues Rochec,d, Pierre Bouzate,f, François Kerbaulc,g, Ugo Scemamah, \\ Thierry Bège ${ }^{c, i, j}$, Pascal Alexandre Thomas ${ }^{c, k}$, Xavier Flecher ${ }^{c, 1}$, Emmanuelle Hammada, \\ Marc Leone ${ }^{\mathrm{a}, \mathrm{c}, \mathrm{m}}$
}

${ }^{a}$ Department of Anesthesiology and Critical Care, North university hospital, AP-HM, Marseille, France

${ }^{\mathrm{b}}$ Aix Marseille Univiversity, INSERM, INRA, C2VN, Marseille, France

${ }^{c}$ Aix-Marseille University, Marseille, France

d Department of Neurosurgery, North university hospital, AP-HM, Marseille, France

e Department of Anesthesiology and Intensive Care Medicine, Grenoble University Hospital, Grenoble, France

${ }^{\mathrm{f}}$ Grenoble Alps University, Grenoble, France

${ }^{\mathrm{g}}$ Pole RUSH, Assistance-Publique Hôpitaux de Marseille, Marseille, France

${ }^{\mathrm{h}}$ Department of Radiology, Hôpital Nord, Marseille, France

${ }^{\mathrm{i}}$ Department of General Surgery, Marseille, France

${ }^{j}$ Laboratoire de Biomécanique Appliquée, UMR T24, Marseille, France

${ }^{\mathrm{k}}$ Department of Thoracic and Esophageal Surgery, Hôpital Nord, Marseille, France

${ }^{1}$ Department of Orthopaedic Surgery, Institut du Mouvement et de l'Appareil Locomoteur, UMR CNRS 7287 /AMU, Centre Hospitalo-Universitaire Sud, Hôpital Sainte Marguerite, Marseille, France

${ }^{\mathrm{m}}$ Unité de Recherche sur les Maladies Infectieuses et Tropicales Emergentes, UMR 63, Marseille, France

\section{A R T I C LEINFO}

\section{Keywords:}

Point of care ultrasound

Whole body computed tomography

Trauma bay

Severe trauma

Diagnostic accuracy

Chest x-ray

Pelvic x-ray

Focused assessment with sonography for trauma

\begin{abstract}
A BSTR A C T
Background: Before total body computed tomography scan, an initial rapid imaging assessment should be conducted in the trauma bay. It generally includes a chest x-ray, pelvic x-ray, and an extended focused ultrasonography assessment for trauma. This initial imaging assessment has been poorly described since the increase in the use of ultrasound. Therefore, our study aimed to evaluate the diagnostic accuracy and therapeutic impact of this initial imaging work-up in severe trauma patients. A secondary aim was to assess the therapeutic impact of a chest x-ray according to the lung ultrasonography findings.

Methods: Patients with severe trauma who were admitted directly to our level 1 trauma center were consecutively included in this retrospective single center study. The diagnostic accuracy, therapeutic impact, and appropriate decision rate were calculated according to the initial assessment results of the whole body computed tomography scan and surgery reports.

Results: Among the 1315 trauma patients admitted, 756 were included in this research. Lung ultrasound showed a higher diagnostic accuracy for haemothorax and pneumothorax cases than the chest $x$-ray. Sensitivity and specificity of the abdominal ultrasound to detect intraperitoneal effusion were $70 \%$ and $96 \%$, respectively. The initial assessment had a therapeutic impact in $76(10 \%)$ of the patients, including 16 $(2 \%)$ immediate laparotomies and $58(7 \%)$ chest tube insertions. The pelvic $x$-ray had no therapeutic impact, and when the lung ultrasound was normal, the chest $x$-ray had a therapeutic impact of only $0.13 \%$. Combining the chest $x$-ray and lung ultrasound allowed adequate management of all the pneumothorax and haemothorax cases. Only one of the 756 patients had initial management that was judged as inappropriate. This patient had a missed pelvic disjunction with active retroperitoneal bleeding, and
\end{abstract}



underwent an inappropriate immediate laparotomy.

Conclusions: In our cohort, the initial imaging assessment allowed appropriate decisions in 755 of 756 patients, with a global therapeutic impact of $10 \%$. The pelvic $x$-ray had a minimal therapeutic impact, and in the patients with normal lung ultrasounds, the chest $x$-ray marginally affected the management of our patients. The potential consequences of abandoning systematic chest and pelvic $x$-rays should be investigated in future randomized prospective studies.

(C) 2018 Elsevier Ltd. All rights reserved.

\section{Introduction}

Trauma is one of the leading causes of death worldwide [1]. Some of these deaths are potentially avoidable, requiring improved procedures [2]. According to Advanced Trauma Life Support (ATLS), trauma requires a rapid assessment of injuries and the institution of life-preserving therapy [3]. To date, this approach is based on a chest x-ray (CXR), pelvic x-ray (PXR), and focused assessment with sonography for trauma (FAST) [4]. This initial imaging work-up enabled healthcare practitioners to make appropriate immediate decisions before the use of a whole-body computed tomography (WBCT) scan [5]. Recently, lung ultrasonography (LUS) was added to create an extended FAST (eFAST) in order to assess early thoracic injuries [6].

In trauma patients, an increasing body of literature has shown that the LUS accuracy is superior to that of the CXR [7-9]. However, to our knowledge, no studies investigating the diagnostic accuracy and therapeutic impact of an initial imaging work-up, including a CXR, PXR, and eFAST, have been conducted. In a prior study, Hamada et al. replaced x-rays with the eFAST in stable trauma patients [10], and they showed that this replacement was not associated with missed diagnoses. Our study is the first to evaluate the utility of the CXR and PXR in severe trauma since the introduction of the eFAST in the trauma bay.

This study aimed to evaluate the diagnostic accuracy and therapeutic impact of an initial imaging work-up including a CXR, PXR, and eFAST in severe trauma patients. A secondary aim was to assess the therapeutic impact of the CXR according to the eFAST findings.

\section{Patients and methods}

This retrospective observational study was conducted at the North University Hospital in Marseille, France from August2012 to
August 2017. All the patients with severe trauma who were directly admitted to our trauma center were consecutively included in this research. This study was in compliance with the Strengthening the Reporting of Observational Studies in Epidemiology (STROBE) recommendations [11].

This study was approved by the National Commission for Information Technology and Civil Liberties (authorization no: 2017-38) and by the Ethical Committee of the French Society of Anesthesiology and Critical Care (CERAR, IRB 00010254).

\section{Inclusion and data collection}

All the patients with severe trauma, according to the French trauma triage criteria, who were admitted directly to our trauma center were included in this study [12]. Those patients who were transferred from another hospital or from our emergency department were not included in this research. Patients with missing data greather than $5 \%$ were also excluded. For each patient, the following items were recorded: injury mechanism, demographic features, cardiac arrest on scene, chest decompression on scene, Glasgow Coma Scale (GCS) on scene, and haemodynamic instability at admission (defined as a systolic arterial blood pressure $<90 \mathrm{mmHg}$ or the use of a vasopressor) [13]. Upon admission, the following werecalculated: Abbreviated Injury Scale (AIS), Injury Severity Score (ISS) [14], and Simplified Acute Physiology Score (SAPS II) [15].

\section{Imaging protocol}

Upon admission to the trauma bay, according to our local protocol (Fig. 1), each of the patients underwent a bedside imaging work-up, including a CXR, PXR, and eFAST. The eFAST consisted of an ultrasonographic assessment of the abdomen, pelvis, pericardium, and pleura [16]. It was performed under the direct

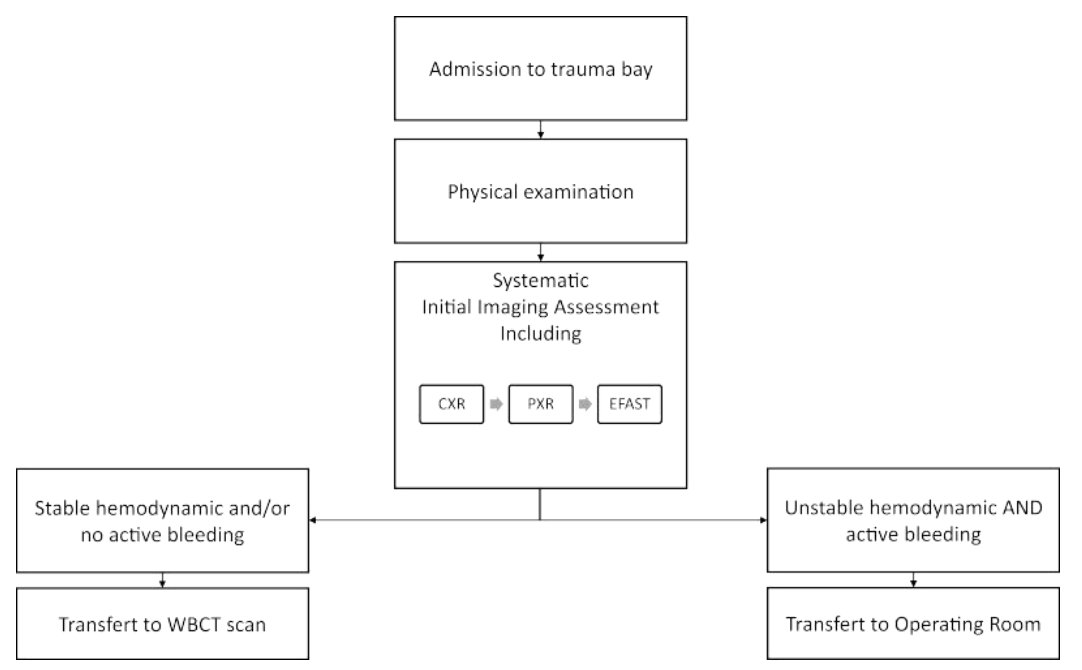

Fig. 1. Institutional protocol.

CXR: chest X-ray; PXR: pelvic x-ray; eFAST: extended focused assessment with sonography for trauma; WBCT: whole-body. computed tomography. 
supervision of the senior intensivist in charge of the patient. The eFAST technique was in compliance with the international guidelines [3].

\section{Diagnostic accuracy}

The results of the initial imaging work-up were prospectively collected in a standardized file. Once stabilized, each patient was transferred to the radiology department where a WBCT scan was performed according to the international guidelines [3]. Those who did not respond to the initial resuscitation underwent an immediate bleeding control procedure before the WBCT scan on the basis of the initial imaging results.

The accuracy of each procedure was compared with the WBCT scan or surgical findings, which served as a reference method. This was expressed using the sensitivity, specificity, positive predictive value, negative predictive value, likelihood ratio, and Youden index $[17,18]$. Occult pneumothorax cases defined as a collection of air less than $1 \mathrm{~cm}$ thick on the largest slice and observed on less than 5 contiguous images were excluded from the statistical analysis [19]. Haemothorax and haemoperitoneum cases in which the radiologist estimated volume was less than $100 \mathrm{ml}$ were also excluded [7].

\section{Therapeutic impact}

Immediate decisions preceding the WBCT scan based on the initial imaging assessment as evaluated by our study were defined as follows:

- CXR and eFAST: immediate chest decompression and/or chest drainage and/or immediate thoracotomy [20].

- Pericardial ultrasonography: pericardial drainage [21].

- Abdominal ultrasonography: laparotomy [3].

- PXR: pelvic angiography and possible embolization [22].

The therapeutic impact of each imaging technique was defined as the following ratio: number of therapeutic changes induced by an imaging technique related to the total number of imaging techniques.

\section{Appropriate decision}

The decision associated with each initial imaging procedure was a posteriori classified as appropriate or inappropriate by 2 investigators (LZ and RF). They analyzed the patient's file, including the radiological and surgical reports. When discordance occurred between the 2 investigators, a third investigator (ML) made the final decision. For the CXR and LUS, the following decisions were considered to be appropriate:

- Immediate pleural decompression or chest tube insertion in the case of life-threatening conditions (haemodynamic instability and/or respiratory distress) associated with a compressive pneumothorax, haemothorax, or haemopneumothorax (including patients with a pneumothorax or haemothorax on the LUS but absent on the CXR) [20].

- Chest tube insertion in patients with a pneumothorax present on the CXR and LUS without life-threatening conditions.

For the pericardial ultrasonography, the following was considered appropriate: drainage of the pericardial fluid in the case of clinical and/or ultrasound signs of tamponade, as defined by the guidelines of the European Society of Cardiology for the diagnosis and management of pericardial diseases [21]. The following was considered to be appropriate for the abdominal ultrasonography: the decision to perform an immediate laparotomy in order to control bleeding in the case of free peritoneal effusion in the abdominal ultrasound in an unstable patient, and the presence of a haemoperitoneum at the opening of the peritoneal cavity as confirmed by the surgical reports [3]. Finally, for the PXR, the following was appropriate: the decision to perform direct vascular embolization or peritoneal packing and the presence of active bleeding as confirmed by angiography [22].

Without such indications, abstention was judged as appropriate. In all other cases, the decisions were judged as inappropriate. A decision was defined as positive when an immediate procedure was deemed necessary and negative in the case of therapeutic abstention.

\section{Imaging characteristics}

The radiation exposure levels were $0.05 \mathrm{mSv}$ and $1.2 \mathrm{mSv}$ for the CXR and PXR, respectively [12].

\section{Statistical analysis}

The statistical analysis was performed using the R Project for Statistical Computing 3.3.1 for Linux Ubuntu software (Vienna, Austria) [23]. The data were presented as the number (percentage) for the qualitative variables and median and interquartile range [ $25^{\text {th }} ; 75^{\text {th }}$ percentile] for the quantitative variables. The accuracy of each method in diagnosing traumatic lesions using the WBCT scan as the reference method was expressed using the sensitivity, specificity, positive predictive value, negative predictive value, likelihood ratio, Youden index, and diagnostic accuracy expressed as the following: [(true positive + true negative)/(true posi- tive + true negative + false positive + false negative)] [24]. The independence between the right and left chest walls for the occurrence of each thoracic lesion was tested using Fisher's exact test [7].

\section{Results}

Between August 2012 and August 2017, 1315 severe trauma patients were admitted to our trauma center. Of these, 756 were admitted directly to the trauma bay, including 690 (90\%) blunt trauma patients (Fig. 2). The mean age was 37 [23; 49] years old and $620(82 \%)$ patients were male. The cause of the blunt trauma was a motor accident in $526(70 \%)$ cases, and the median time spent in the trauma bay was $24[15 ; 37]$ min (Table 1$)$.

\section{$X$-rays}

At admission, CXRs were performed in 745 (99\%) patients. The diagnostic accuracy of the CXR is shown in Table 2, and it had a therapeutic impact for $53(7 \%)$ patients. Of these, 41 pneumothorax and 13 haemothorax cases were treated on the basis of the CXR and LUS findings. No haemothorax cases were treated based only on the CXR findings. Five pneumothorax cases were treated on the basis of abnormal CXR results only and immediate life-threatening conditions, according to our protocol. In these cases, the LUSdid not contribute to the diagnosis due to subcutaneous emphysema. The specific therapeutic impact of the CXR is shown in Table 3. At admission, PXRs were performed in 745 (99\%) patients; however, the PXR had no therapeutic impact. The specific therapeutic impacts of the PXR and CXR are shown in Table 3.

\section{Lung ultrasonography}

At admission, LUSs were performed in 751 (99\%) patients, and the diagnostic accuracy of the LUS is shown in Table 2. The LUS had a therapeutic impact for $53(7 \%)$ patients, including 41 


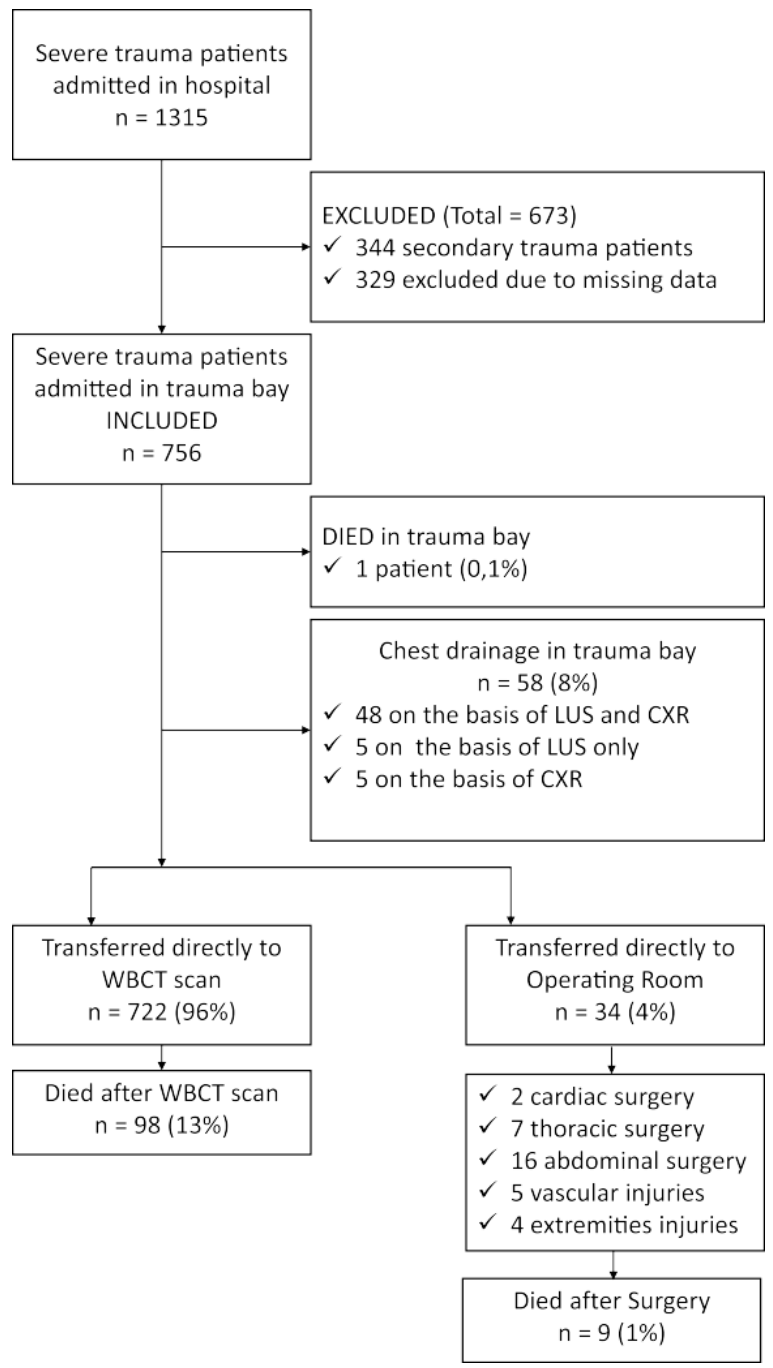

Fig. 2. Flow chart of the study.

LUS: lung ultrasonography; CXR: chest x-ray; WBCT: whole-body computed tomography.neurosurgery, North university

pneumothorax and 13 haemothorax cases. Nine haemothorax cases were treated only on the basis of the LUS. Eight out of these 9 patients had abnormal LUSs and immediate life-threatening conditions. Six pneumothorax cases were treated on the basis of abnormal LUS and immediate life-threatening conditions. The specific therapeutic impact of the LUS is shown in Table 3.

\section{Pericardial ultrasonography}

Pericardial ultrasonography was performed in 696 (92\%) patients. Three $(0.4 \%)$ patients had pericardial effusion, and all of them had penetrating chest traumas. One patient was stable and underwent a WBCT scan, which overturned the pericardial effusion diagnosis. Two of the patients underwent immediate surgical drainage due to clinical signs of tamponade. The diagnoses were a pericardial wound and a left ventricular wound. The specific therapeutic impact of the pericardial ultrasonography is shown in Table 3.

\section{Abdominal ultrasonography}

Abdominal ultrasound examination was performed in 756 $(100 \%)$ patients, and peritoneal effusion was found with the
Table 1

Demographic and clinical characteristics of the patients at the time of initial evaluation.

\begin{tabular}{ll}
\hline & Patients (n= 756) \\
\hline Blunt trauma & $690(91.3 \%)$ \\
Injury type & \\
Motor accident & $526(69.7 \%)$ \\
Fall & $123(16.3 \%)$ \\
Sports-related & $13(1.7 \%)$ \\
$\quad$ Other & $94(12.4 \%)$ \\
Age (years) & $37[23 ; 49]$ \\
Male sex & $620(82.0 \%)$ \\
In-hospital mortality & $108(14.3 \%)$ \\
SAPS II & $33[21 ; 50]$ \\
ISS & $25[16 ; 34]$ \\
Cardiac arrest & $35(4.6 \%)$ \\
Haemodynamic instability & $257(34.0 \%)$ \\
Norepinephrine infusion & $165(21.8 \%)$ \\
Catheters before WBCT & $506(66.9 \%)$ \\
Brain trauma & $374(49.5 \%)$ \\
GCS score & $10[7 ; 15]$ \\
Chest trauma & $430(56.9 \%)$ \\
Mechanical ventilation & $378(50.0 \%)$ \\
Prehospital chest tube & $20(2.6 \%)$ \\
Subcutaneous emphysema & $66(8.7 \%)$ \\
\hline
\end{tabular}

Values shown are $\mathrm{n}(\%)$ or median $\left[25^{\text {th }} ; 75^{\text {th }}\right.$ percentile]. SAPS II Simplified Acute Physiology Score, ISS Injury Severity Score, WBCT whole-body computed tomography, GCS Glasgow Coma Scale.

abdominal ultrasound in $106(14 \%)$ patients. The diagnostic accuracy of the abdominal ultrasound is shown in Table 2. A therapeutic impact (immediate laparotomy) was found in $16(2 \%)$ of the patients. Active peritoneal bleeding was found in 13 of the 16 $(81 \%)$ patients. Two $(12 \%)$ patients had no active bleeding (liver wounds), and 1 (7\%) patient had a retroperitoneal haemorrhage due to a pelvic arterial wound, without any abdominal injury. The specific therapeutic impact of the abdominal ultrasonography is shown in Table 3.

\section{Decision appropriateness}

Among the 756 patients, $76(10 \%)$ required at least 1 immediate treatment. The global therapeutic impact of the initial imaging work-up was $10 \%$, and was considered to be appropriate in $75(99 \%)$ patients.

Theoretically, the use of a CXR or LUS alone would have generated an inappropriate decision in $15(2 \%)$ patients. Indeed, 14 clinically relevant haemothorax cases would have been misdiagnosed with the sole use of a CXR, and 1 clinically relevant pneumothorax case would have been misdiagnosed with the sole use of an LUS. However, in clinical practice, the combination of a CXR and LUS resulted in an appropriate therapeutic decision for all the chest drainage cases. Therefore, all the clinically relevant pneumothorax and haemothorax cases were adequately treated before the WBCT scan.

Immediate laparotomies were performed in $16(2 \%)$ patients. In 1 case, a laparotomy was judged to be inappropriate because the haemodynamic instability was due to active retroperitoneal bleeding. The PXR of this patient performed in the trauma bay showed a pubic symphysis disjunction. This patient had pre peritoneal packing and vascular embolization immediately after the inappropriate laparotomy. However, he should have been oriented to undergo vascular embolization or peritoneal packing before the laparotomy. Among the 756 patients, only one other case required an urgent vascular embolization procedure for pelvic trauma before performing a WBCT scan due to an open pelvic trauma with active external bleeding. All the 39 others vascular embolization were performed after the WBCT. 
Table 2

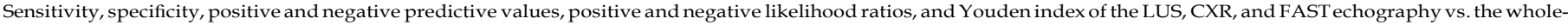
body computed tomography (WBCT) scan for detecting traumatic lesions.

\begin{tabular}{|c|c|c|c|c|c|c|c|c|}
\hline & Sensitivity \% & Specificity \% & Youden index & $\begin{array}{l}\text { Positive } \\
\text { predictive } \\
\text { value } \%\end{array}$ & $\begin{array}{l}\text { Negative } \\
\text { predictive } \\
\text { value } \%\end{array}$ & $\begin{array}{l}\text { Positive } \\
\text { likelihood ratio }\end{array}$ & $\begin{array}{l}\text { Negative } \\
\text { likelihood } \\
\text { ratio }\end{array}$ & Diagnostic accuracy \\
\hline \multicolumn{9}{|c|}{ Pneumothorax $(\mathrm{n}=198)$} \\
\hline LUS $(n=1495)$ & 69 & 99 & 0.7 & 94 & 96 & 112 & 0.3 & 96 \\
\hline CXR $(n=1488)$ & 37 & 100 & 0.4 & 95 & 91 & 120 & 0.4 & 91 \\
\hline \multicolumn{9}{|c|}{ Haemothorax $(n=103)$} \\
\hline LUS $(n=1495)$ & 48 & 100 & 0.5 & 90 & 97 & 135 & 0.5 & 96 \\
\hline CXR $(n=1488)$ & 29 & 100 & 0.3 & 90 & 95 & 133 & 0.7 & 95 \\
\hline \multicolumn{9}{|c|}{ Peritoneal effusion $(n=116)$} \\
\hline FAST $(n=756)$ & 70 & 96 & 0.7 & 78 & 95 & 19 & 0.3 & 92 \\
\hline
\end{tabular}

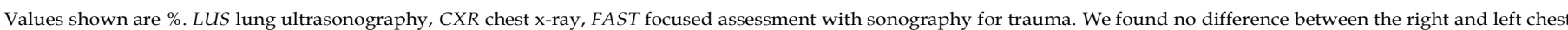

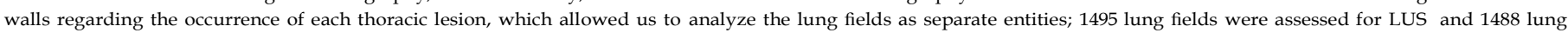
fields for CXR.

Table 3

Specific therapeutic impacts of the different imaging techniques.

\begin{tabular}{|c|c|c|c|c|c|}
\hline Imaging technique & Therapeutic impact & $\begin{array}{l}\text { Appropriate } \\
\text { positive } \\
\text { decision }\end{array}$ & $\begin{array}{l}\text { Inappropriate } \\
\text { positive decision }\end{array}$ & $\begin{array}{l}\text { Appropriate } \\
\text { negative decision }\end{array}$ & $\begin{array}{l}\text { Inappropriate } \\
\text { negative decision }\end{array}$ \\
\hline LUS $(n=751)$ & $5(0.7 \%)$ & $5(100 \%)$ & 0 & $745(99.9 \%)$ & $1(0.1 \%)$ \\
\hline $\mathrm{CXR}(\mathrm{n}=745)$ & $5(0.7 \%)$ & $53(100 \%)$ & 0 & $678(98 \%)$ & $14(2 \%)$ \\
\hline LUS + CXR $(n=741)$ & $48(6 \%)$ & $48(100 \%)$ & 0 & $693(100 \%)$ & 0 \\
\hline Pericardial sonography $(n=683)$ & $2(0.3 \%)$ & $2(100 \%)$ & 0 & $681(100 \%)$ & 0 \\
\hline $\operatorname{PXR}(\mathrm{n}=745)$ & 0 & 0 & 0 & $744(99.9 \%)$ & $1(0.1 \%)$ \\
\hline Abdominal sonography $(\mathrm{n}=756)$ & $16(2 \%)$ & $15(94 \%)$ & $1(6 \%)$ & $740(100 \%)$ & 0 \\
\hline Global therapeutic impact of initial assessment & $76(10 \%)$ & $123(99.2 \%)$ & $1(0.8 \%)$ & 741 (99.6\%) & $1(0.4 \%)$ \\
\hline
\end{tabular}

Values shown are $\mathrm{n}(\%)$. LUS lung ultrasonography, CXR chest x-ray, FAST focused assessment with sonography for trauma.

\section{Imaging characteristics}

The global radiation was $952 \mathrm{mSv}$ (745 CXR and 745 PXR).

\section{Discussion}

Our study showed that an initial imaging assessment was associated with a rate of $755 / 756$ appropriate decisions. It also underscored the low therapeutic impact of a PXR and CXR, especially when the LUS is normal.

As expected, for the pneumothorax and haemothorax cases, the diagnostic accuracy of the LUSwashigher than that of theCXR [7-9]. However, ourfindingsshowed that therate of appropriate decisions was higher with the LUS than with the CXR [5]. When the LUS and CXR were combined, the rate of appropriate decisions was $100 \%$. Before the development of eFAST, Peytel et al. missed 2 clinically relevant pneumothorax cases among 400 patients using the CXR alone. Indeed, our study confirms the benefits of implementing an LUS in the initial assessment of trauma patients.

For the first time, we investigated the therapeutic impact of the CXR in combination with the LUS. Interestingly, in the patients with normal LUS results, the diagnostic impact of the CXR was only $0.13 \%$. Recently, with the increasing use of the LUS in the trauma bay, the usefulness of the CXR has been questioned. For example, Hamada et al. showed that, in trauma patients with haemodynamic stability, the exclusion of the CXR and PXR from the initial imaging work-up was feasible and safe [10]. In addition, they questioned the performance of the CXR and PXR in unstable trauma patients. In our cohort, 257 (34\%) patients were considered to be unstable according to our criteria [13], and the PXR findings did not seem to affect their orientations. Our results also suggest that a systematic PXR may be an ineffective procedure in the initial work-up, although it can help in the global assessment. In our cohort, all the trauma patients requiring pelvic vascular embolizations underwent WBCT scans before the procedure. We estimated that, in our cohort, this change of practice would decrease the radiation dosage by $932 \mathrm{mSv}$ [12].

In contrast, there is still a debate around the use of the CXR. In routine practice, the CXR helps in assessing the pneumothorax volume. As previously reported, the LUS only serves to obtain a semiquantification of the pneumothorax [8]. Indeed, the lung point determination in each intercostal space discriminates a minor from a significant pneumothorax. However, in case of complete pneumothorax (defined as a complete separation of the lung from the chest wall), the lung point is absent. In fact, a pneumothorax cannot be evaluated perfectly by an LUS since air stops the ultrasound waves. In contrast, the CXR has a low sensitivity but high specificity in the detection of an anterolateral pneumothorax. Thus, in our daily practice, before the WBCT scan, we perform a chest tube insertion in those patients with pneumothoraces that are discernible on the CXR. In those patients with pneumothoraces on the LUS that are not visible on the CXR, a chest tube was inserted in the case of haemodynamic or respiratory failure that was not explained by another evident cause. Finally, in case of subcutaneous emphysema, ultrasounds cannot reach the pleura and therefore LUS shouldn't be used.

The abdominal ultrasound exhibited good diagnostic accuracy for detecting peritoneal effusion [4]. However, the low therapeutic impact of the abdominal ultrasound and the high rate of active bleeding in the patients who underwent an immediate laparotomy were striking. Our immediate laparotomy rate was 5-fold lower than those reported elsewhere [5]. In our cohort, the rate of patients undergoing immediate laparotomies and in whom active bleeding was found was $81 \%$, while Charbit et al. found a rate of 
$50 \%$ [25]. We explain this difference by the greater use of the WBCT scan in our patients. Studies have shown a survival benefit if the CT scanner is located less than $50 \mathrm{~m}$ from the trauma bay, as in our hospital [26]. In addition, Huber-Wagner et al. suggested a survival benefit with a WBCT scan in patients exhibiting shock [27]. Our hypothesis for our results is that we performed an immediate laparotomy only in the most severe cases, explaining the low incidence of this process.

Our study had several limitations that we must acknowledge. Firstly, it was a retrospective study. However, our data were prospectively collected in order to evaluate our practices, which probably minimized the risk of bias. Consequently, we had to exclude all the cases in which the prospective file containing the results of the initial imaging work-up was not available. Secondly, our study was performed in a single center with a blunt trauma rate above $90 \%$, reducing the extrapolation of our findings. Finally, we focused on the initial imaging work-up, and we did not assess the morbidity and mortality. Therefore, we cannot make conclusions about the associations between the initial imaging workup and the outcome.

\section{Conclusion}

In this observational study, we observed a high rate of appropriate decisions after the initial imaging work-up, including a PXR, CXR, and eFAST. However, the PXR had a minimal therapeutic impact. In those patients with a normal LUS, the CXR marginally affected the management of our patients. The potential consequences of abandoning systematic CXR, and favoring a LUS based strategy involving or not involving a CXR before the WBCT should be investigated in future randomized prospective studies.

\section{Authors' contributions}

Conception and design of the study: LZ.

Acquisition of data: RF.

Analysis and interpretation of data: LZ, RF, FA.

Drafting the article: RF, LZ.

Final approval of the version to be submitted: ML.

\section{Role of the funding source}

This study was supported by institutional and departmental funds.

\section{Ethics approval and consent to participate}

This study was approved by the National Commission for Information Technology and Civil Liberties (authorization no: 2017-38) and by the Ethical Committee of the French Society of Anesthesiology and Critical Care (CERAR, IRB 00010254).

\section{Conflict of interests}

The authors have no conflicts of interest linked to this work to declare.

\section{Acknowledgements}

We greatly appreciated the help of Gary Duclos, Calypso Mathieu, Sophie Medam, Coralie Vigne, Marion Poirier and Emmanuelle Hammad for recruited patients. We appreciated the constructive discussion with Pierre-Hugues Roche, Pierre Bouzat, François Kerbaul, Ugo Scemama, Thierry Bège, Pascal Alexandre Thomas and Xavier Flecher leading to the idea of study.

\section{References}

[1] Sethi D., Racioppi F, Baumgarten I, Vida P. Injuries and violence in Europe: why they matter and what can be done. Inj Violence Eur Why They Matter What Can Be Done 2006.

[2] Cayten CG, Stahl WM, Agarwal N, Murphy JG. Analyses of preventable deaths by mechanism of injury among 13,500 trauma admissions. Ann Surg 1991;214:510-21.

[3] Advanced Trauma Life Support. Am Coll Surg n.d., 2017, https://www.facs.org/ quality \%20programs/trauma/atls [Accessed March 9, 2017]

[4] Scalea TM, Rodriguez A, Chiu WC, Brenneman FD, Fallon WF, Kato K, et al. Focused assessment with sonography for trauma (FAST): results from an international consensus conference. J Trauma 1999;46:466-72.

[5] Peytel E, Menegaux F, Cluzel P, Langeron O, Coriat P, Riou B. Initial imaging assessment of severe blunt trauma. Intensive Care Med 2001;27:1756-61, doi: http://dx.doi.org/10.1007/s00134-001-1119-z.

[6] Volpicelli G, Elbarbary M, Blaivas M, Lichtenstein DA, Mathis G, Kirkpatrick AW, et al. International evidence-based recommendations for point-of-care lung ultrasound. Intensive Care Med 2012;38:577-91, doi:http:/ / dx.doi.org/ $10.1007 / \mathrm{s} 00134-012-2513-4$.

[7] Hyacinthe A-C, Broux C, Francony G, Genty C, Bouzat P, Jacquot C, et al Diagnostic accuracy of ultrasonography in the acute assessment of common thoracic lesions after trauma. Chest 2012;141:1177-83, doi:http://dx.doi.org/ 10.1378/chest.11-0208.

[8] Soldati G, Testa A, Sher S, Pignataro G, La Sala M, Silveri NG. Occult traumatic pneumothorax: diagnostic accuracy of lung ultrasonography in the emergency department. Chest 2008;133:204-11, doi:http://dx.doi.org/10.1378/chest.071595.

[9] Lichtenstein D, Goldstein I, Mourgeon E, Cluzel P, Grenier P, Rouby J-J. Comparative diagnostic performances of auscultation, chest radiography, and lung ultrasonography in acute respiratory distress syndrome. Anesthesiology 2004;100:9-15.

[10] Hamada SR, Delhaye N, Kerever S, Harrois A, Duranteau J. Integrating eFAST in the initial management of stable trauma patients: the end of plain film radiography. Ann Intensive Care 2016;6(62), doi:http://dx.doi.org/10.1186/ s13613-016-0166-0.

[11] von Elm E, Altman DG, Egger M, Pocock SJ, Gøtzsche PC, Vandenbroucke JP, et al. The strengthening the reporting of observational studies in epidemiology (STROBE) statement: guidelines for reporting observational studies. J Clin Epidemiol 2008;61:344-9， doi:http://dx.doi.org/10.1016/i. clinepi.2007.11.008.

[12] IRSN - Doses délivrées aux patients en scanographie et en radiologie conventionnelle - Résultats d'une enquête multicentrique en serveur public IRSN- Rapport-dosimetrie-patient-2010-12.pdf. (n.d.), 2016.

[13] Rossaint R, Bouillon B, Cerny V, Coats TJ, Duranteau J, Fernández-Mondéjar E, et al. The European guideline on management of major bleeding and coagulopathy following trauma: fourth edition. Crit Care Lond Engl 2016;20 (100), doi:http:/ / dx.doi.org/10.1186/s13054-016-1265-x.

[14] Baker SP, O'Neill B, Haddon W, Long WB. The injury severity score: a method for describing patients with multiple injuries and evaluating emergency care. J Trauma 1974;14:187-96.

[15] Le Gall JR, Lemeshow S, Saulnier F. A new simplified acute physiology score (SAPS II) based on a European/North American multicenter study. JAMA 1993;270:2957-63

[16] Kirkpatrick AW, Sirois M, Laupland KB, Liu D, Rowan K, Ball CG, et al. Hand-held thoracic sonography for detecting post-traumatic pneumothoraces: the extended focused assessment with sonography for trauma (EFAST). J Trauma 2004;57:288-95.

[17] Hajibandeh S, Hajibandeh S. Systematic review: effect of whole-body computed tomography on mortality in trauma patients. J Inj Violence Res 2015;7:64-74, doi:http://dx.doi.org/10.5249/jivr.v7i2.613.

[18] Huber-Wagner S, Lefering R, Qvick L-M, Körner M, Kay MV, Pfeifer K-J, et al. Effect of whole-body CT during trauma resuscitation on survival: a retrospective, multicentre study. Lancet Lond Engl 2009;373:1455-61, doi: http://dx.doi.org/10.1016/S0140-6736(09)60232-4.

[19] Wolfman NT, Myers WS, Glauser SJ, Meredith JW, Chen MY. Validity of CT classification on management of occult pneumothorax: a prospective study. AJR Am J Roentgenol 1998;171:1317-20, doi:http:// dx.doi.org/10.2214/. ajr.171.5.9798871

[20] Bouzat P, Raux M, David JS, Tazarourte K, Galinski M, Desmettre T, et al. Chest trauma: first 48hours management. Anaesth Crit Care Pain Med 2017;36:13545, doi:http://dx.doi.org/10.1016/j.accpm.2017.01.003.

[21] Adler Y, Charron P, Imazio M, Badano L, Barón-Esquivias G, Bogaert J, et al. 2015 ESC guidelines for the diagnosis and management of pericardial diseases: the task force for the diagnosis and management of pericardial diseases of the European society of cardiology (ESC)endorsed by: the European Association for Cardio-Thoracic Surgery (EACTS). Eur Heart J 2015;36:2921-64, doi:http:/ / dx.doi.org/10.1093/eurheartj/ehv318.

[22] Coccolini F, Stahel PF, Montori G, Biffl W, Horer TM, Catena F, et al. Pelvic trauma: WSES classification and guidelines. World J Emerg Surg WJES 2017;12:, doi:http:/ /dx.doi.org/10.1186/s13017-017-0117-6.

[23] R: The R Project for Statistical Computing (n.d.),2017, https:/ / www.r-project. org/ [Accessed 10 March 2017].

[24] Jaeschke R, Guyatt GH, Sackett DL. Users' guides to the medical literature. III. How to use an article about a diagnostic test. B. What are the results and will 
they help me in caring for my patients? The Evidence-Based Medicine Working Group. JAMA 1994;271:703-7.

[25] Charbit J, Millet I, Lakhal K, Brault-Noble G, Guillon F, Taourel P, et al. A haemoperitoneum does not indicate active bleeding in the peritoneum in 50\% of hypotensive blunt trauma patients: a study of 110 severe trauma patients. Injury 2014;45:88-94, doi:http:/ / dx.doi.org/10.1016/j.injury.2012.05.018.

[26] Huber-Wagner S, Mand C, Ruchholtz S, Kühne CA, Holzapfel K, Kanz K-G, et al. Effect of the localisation of the CT scanner during trauma resuscitation on survival - a retrospective, multicentre study. Injury 2014;45(Suppl. 3):S76-82, doi:http:/ / dx.doi.org/10.1016/j.injury.2014.08.022.

[27] Huber-Wagner S, Biberthaler P, Häberle S, Wierer M, Dobritz M, Rummeny E, et al. Whole-body CT in haemodynamically unstable severely injured patients-a retrospective, multicentre study. PloS One 2013;8:e68880, doi: http:/ / dx.doi.org/10.1371/journal.pone.0068880. 